associated with a high likelihood of complete cytoreduction (93\%) compared to a PCI of greater than 20, where complete cytoreduction was achieved in the minority (44\%). Assessment and validation of PCI by radiology, laparoscopy and laparotomy may help in the selection of patients for cytoreductive surgery, neoadjuvant chemotherapy or chemotherapy alone. Disclosures None.

\section{POSTOPERATIVE OUTCOMES OF PRIMARY AND INTERVAL CYTOREDUCTIVE SURGERY FOR ADVANCED OVARIAN CANCER REGISTERED IN THE DUTCH GYNECOLOGICAL ONCOLOGY AUDIT (DGOA)}

${ }^{1}$ Nishita Baldewpersad Tewarie, ${ }^{2}$ Willemien JVan Driel, ${ }^{3}$ Maaike Van Ham, ${ }^{4}$ Michel Wouters, ${ }^{5}$ Roy FPM Kruitwagen. ${ }^{1}$ Radboud University Medical Center; ${ }^{2}$ Netherlands Cancer Institute, Department of Gynecology; ${ }^{3}$ Radboud University Medical Center, Department of Obstetrics and Gynecology, Nijmegen, The Netherlands; ${ }^{4}$ Netherlands Cancer Institute, Department of Surgical Oncology, Amsterdam, The Netherlands; ${ }^{5}$ Maastricht University Medical Centre (Mumc), Department of Obstetrics and Gynecology, and Grow-School for Oncology and Developmental Biology, Maastricht, The Netherlands

\subsection{6/ijgc-2020-ESG0.152}

Introduction/Background The challenge when performing cytoreductive surgery (CRS) for advanced ovarian cancer is to balance the benefits (obtaining complete CRS) and risks (perioperative complications). The aim of this study was to report short term postoperative morbidity and mortality in relation to surgical outcome in patients undergoing primary cytoreductive surgery(PCS) and/or interval cytoreductive (ICS) surgery in 8 gynaeco-oncological regions in the Netherlands.

Methodology Data from the prospective Dutch Gynecological Oncology Audit(DGOA) data base were used for this retrospective analysis with population-based data. All patients with advanced ovarian cancer (FIGO IIB-V) undergoing PCS or ICS between January 1st, 2015- December 31st, 2018 were included. Primary outcome was the frequency of postoperative complications. In addition, median time to adjuvant chemotherapy was shown in relation to CRS outcomes and complication severity. Hospitals were clustered in 8 regions consisting of a gynaeco-oncological center and its referring hospitals. Complications with Clavien Dindo $\geq 3$ were analyzed per region and casemix corrected.

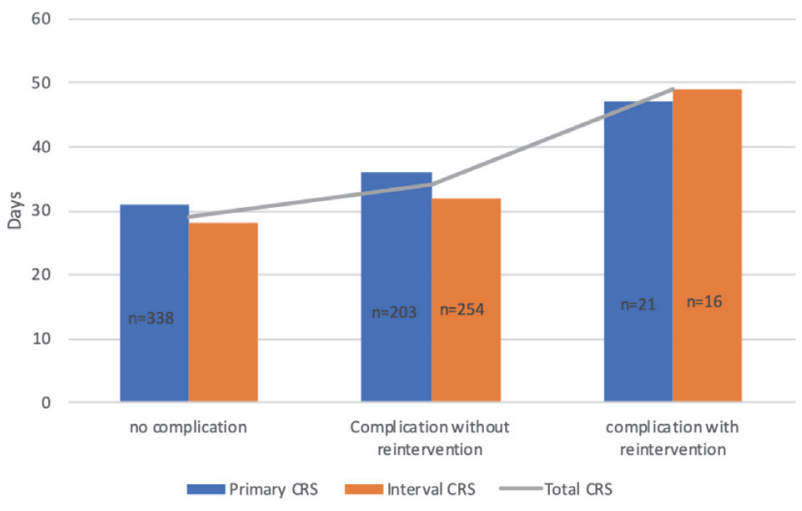

Abstract 565 Figure 2c Median waiting time for adjuvant chemotherapy after complete cytoreduction (days)

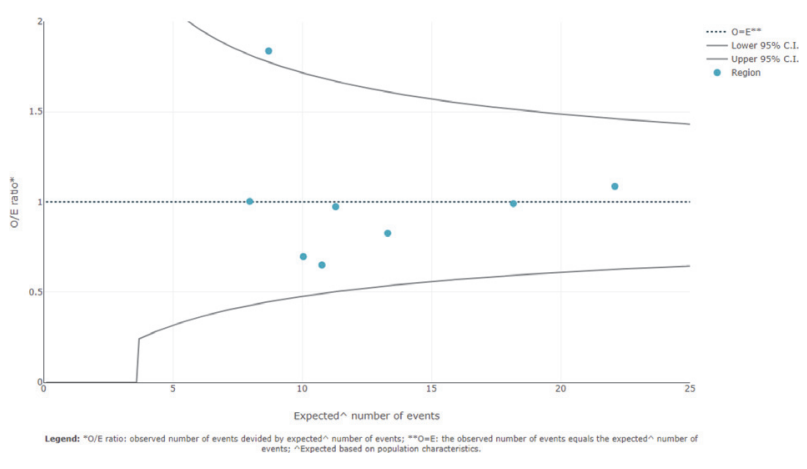

Abstract 565 Figure 3a Casemix corrected funneplot for patients with Clavien Dindo $>3$ in Primary CRS for 8 regions in the Netherlands

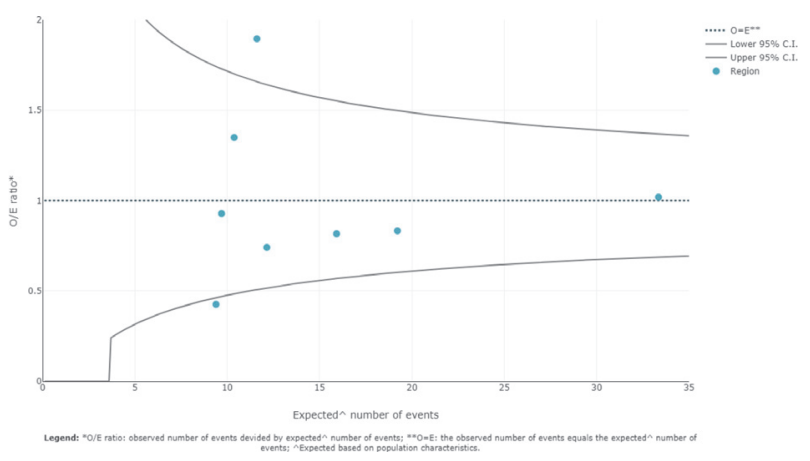

Abstract 565 Figure 3b Casemix corrected funnelplot for patients with clavien Dindo $>3$ in Interval CRS for 8 regions in the Netherlands

Results A total of 2382 patients met the inclusion criteria corresponding to 2458 surgical procedures. 1027 patients underwent PCS and 1355 patients underwent ICS, a third group contained patients with both PCS and ICS $(n=76)$. Complications with re-invention were significantly higher in PCS compared to ICS $(5.7 \%$ vs. $3.6 \%$ respectively, p $=0.048)$, but complete CRS was achieved more often in PCS compared to ICS $(69.7 \%$ vs $62.1 \%$ respectively<0.001). Clavien Dindo $\geq 3$, ICU stay, and 30 -day mortality were not statistically different between PCS and ICS. Time to adjuvant chemotherapy was the longest in patients with complete CRS and a complication with reintervention:47 days (figure 2c). Regional variation for Clavien Dindo $\geq 3$ was apparent with 1 region as outlier in PCS and ICS (figure $3 \mathrm{a}$ and $3 \mathrm{~b}$ ).

Conclusion Complete PCS is more often achieved, but there are more complications with re-intervention resulting in more time to start with adjuvant chemotherapy. This exceeds the advised maximum of 42 days. This finding underlines the importance of maintaining a balance in aggressiveness of surgery and result of CRS in relation to survival. In addition, complications in the Netherlands show regional hospital variation after casemix correction.

In the future these outcomes should be discussed to minimize complications and therefore improve quality of care within the nation.

Disclosures None. 